\title{
Cardiac biomarkers in acute coronary syndromes
}

\section{Naresh Ranjith and Datshana P. Naidoo}

Department of Medicine, Coronary Care Unit, R. K. Khan Hospital, Inkosi Albert Luthuli Central Hospital

Address for correspondence:

Dr N. Ranjith

Coronary Care Unit

R. K. Khan Hospital

Private Bag X004

Chatsworth

4030

Durban

KwaZulu-Natal

Email:

ranjith@lantic.co.za

\section{ABSTRACT Cardiac biomarkers play a significant} role in the diagnosis, risk assessment, and management of patients with acute coronary syndromes [ACS]. Several biomarkers identify different components of the pathophysiology of ACS: troponins are markers of myocyte necrosis, natriuretic peptides reflect neurohormonal activation and myocardial dysfunction, and C-reactive protein reflects various inflammatory processes. Whilst there are a number of established and novel biomarkers to assess ischemia, necrosis and myocardial dysfunction in coronary artery disease, it is probable that no single biomarker will emerge that provides appropriate information for all clinical settings of ACS. This suggests that ongoing efforts in plasma-based biomarker research should concentrate on the use of a multimarker approach to enhance our diagnosis, prognostic assessment, and management of patients with suspected ACS, as compared with using individual markers alone.

It is recommended that current practice involve the selective use of biomarkers in patients in whom a more complete assessment of risk is desired. At present, routine biomarker measurement is not advocated until further data become available, which will support the benefit of specific therapeutic interventions. SAHeart 2008; 5:70-75

\section{INTRODUCTION}

Ischemic heart disease is the number one cause of mortality and morbidity in the Western world, and this unfortunate reality is unlikely to change in the future. In fact, it is believed that in 2020 acute coronary syndromes (ACS) will over-run infectious disease and cancer to become the leading cause of death and disability, not only in industrialized countries, but also in the developing world.(1) However, despite these facts the diagnostic approach to ACS remains one of our most difficult and controversial challenges. ${ }^{(2)}$

As our understanding of the pathobiology of atherothrombosis has improved, researchers have attempted to evaluate the activities of these biological processes by measuring markers in plasma or urine (i.e. biomarkers). Indeed, several conventional and promising new markers of myocardial injury have been identified.

This overview focuses on established and emerging biomarkers and their role in the diagnosis, risk stratification, and prognostication of patients presenting with ACS.

\section{HISTORICAL BACKGROUND TO THE DIAGNOSIS OF ACUTE CORONARY SYNDROMES}

In 1971, the World Health Organization (WHO) developed a definition of acute myocardial infarction (AMI), which requires the presence of 2 of the following 3 major criteria: chest pain suggestive of myocardial ischemia, electrocardiographic (ECG) manifestations and indicative laboratory changes. ${ }^{(3)}$ This was a major innovation, and by the early 1990s the WHO definition had become a cornerstone in clinical and laboratory medicine.

Despite increasing focus on biochemical markers during the last 2 decades, the search for the optimal marker is ongoing. Early markers of AMI such as aspartate aminotransferase, lactate dehydrogenase and creatine kinase (CK), were non-specific and did not provide definitive proof of myocardial involvement. Assays to measure the enzymatic activity of the creatine kinase muscle brain isoenzyme (CK-MB) was an important advancement, especially in terms of improved specificity. ${ }^{(4)}$ Although CK-MB is not ideal for early risk stratification, it became the standard against which new biochemical markers are compared with respect to diagnostic accuracy and quantification of myocardial necrosis. 
By the mid-1990s, cardiospecific troponins were identified as effective markers of myocardial injury. On the basis of their improved sensitivity and greater tissue-specificity, troponins have evolved into our most important diagnostic markers, becoming the biochemical "gold standard" for the diagnosis of myocardial injury in patients with acute chest pain. This led to the realization that the definition of AMI needed to be revisited.

In 2000, a joint European Society of Cardiology and American College of Cardiology Committee updated the definition of AMI, identifying troponins as the cardiac markers of choice. ${ }^{(5)}$ More recently, this committee has proposed the following definition of AMI:

Typical rise and / or gradual fall of troponin (and measurements of CK-MB only to be performed if troponin assays are not available), with at least one of the following:

a. Ischemic symptoms

b. Development of pathologic Q waves on the ECG

c. ECG changes indicative of ischemia (ST-segment elevation or depression)

d. Coronary artery intervention (e.g. angioplasty)

Pathologic findings of an AMI

\section{CRITERIA FOR BIOMARKER EVALUATION IN ACS}

Morrow and De Lemos have proposed that evaluation of the clinical utility of new biomarkers requires at least 3 fundamental questions to be answered. ${ }^{(6)}$

\section{Can the clinician measure the biomarker?}

The biomarker must be easy to measure in a cost-effective manner, and not require specialized plasma collection techniques or assay techniques. Available assays should be easily accessible and have a prompt turnaround time.

2. Does the biomarker add new information to the risk assessment?

It is important that the proposed biomarker provide information, which adds to or improves upon existing available tests. A strong and consistent association should exist between the biomarker and patients with ACS with respect to clinical outcome to allow for prognostic application of that marker.

\section{Will the biomarker help the clinician to manage patients?}

As outlined in Figure I, each biomarker has the potential to address different mechanisms in the pathobiology of plaque instability and, therefore, may be of use for the early detection of sub-clinical disease, diagnosis of ACS, risk stratification, selection of appropriate therapy, and monitoring of disease progression. Each of these potential uses merits consideration in the evaluation of a biomarker's ability to enhance the management of patients presenting with ACS.

Several biomarkers meet these criteria, but only few are ready for clinical practice.

\section{TROPONINS}

Cardiac Troponin $\mathrm{T}$ and I are cardiac regulatory proteins that control the calcium-mediated interaction of actin and myosin. ${ }^{(7)}$ Both have cytosolic and structural pools, with most existing in the structural pool. ${ }^{(8)}$ An important advantage of the troponins is that their isoforms are unique to cardiac myocytes, with most of the early troponin release during an AMI coming from the cytosolic pool. Subsequent release is prolonged, with degradation of the actin and myosin filaments in the area of damage.

The diagnostic cut-off for cardiac troponins remains a source of contention. The ESC / ACC consensus document recommended that each laboratory should define its cut-offs for each test at the 99th percentile of a normal reference population, and that this cut-off value should be measurable with a coefficient of variation (CV) of 10 percent or less. ${ }^{(5)}$ However, current assays are unable to detect tropinin in the majority of normal healthy individuals and are imprecise at this low level. Until better assays are available, some have suggested that the

FIGURE I: Clinical Role of Cardiac Biomarkers in ACS

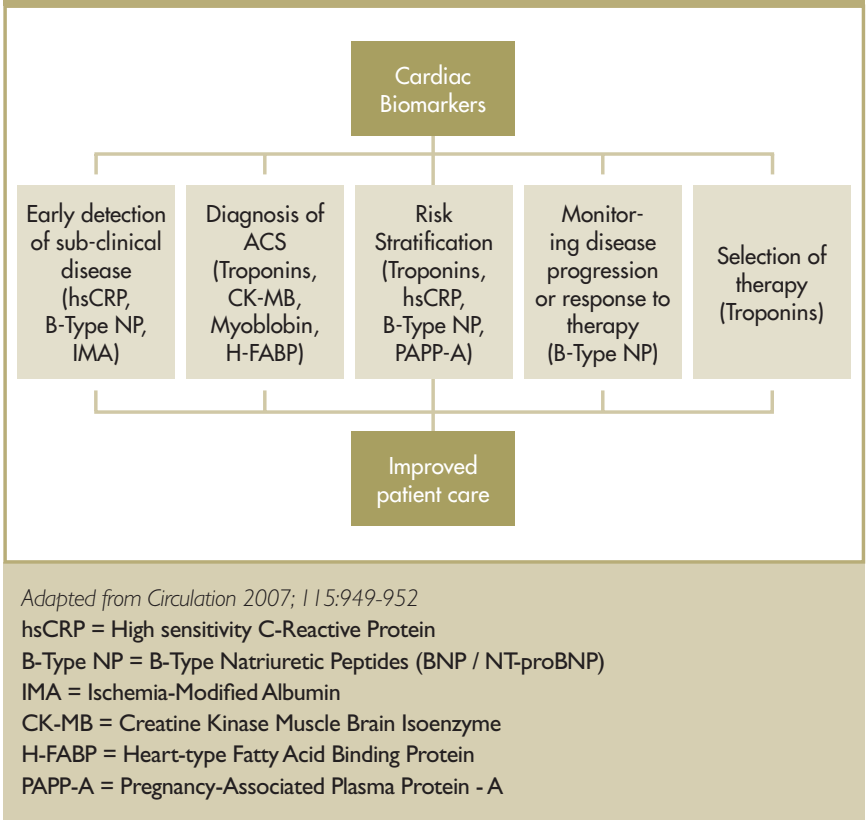


FIGURE 2: Time Course of Troponin and CK-MB in Blood after Onset of Myocardial Infarction

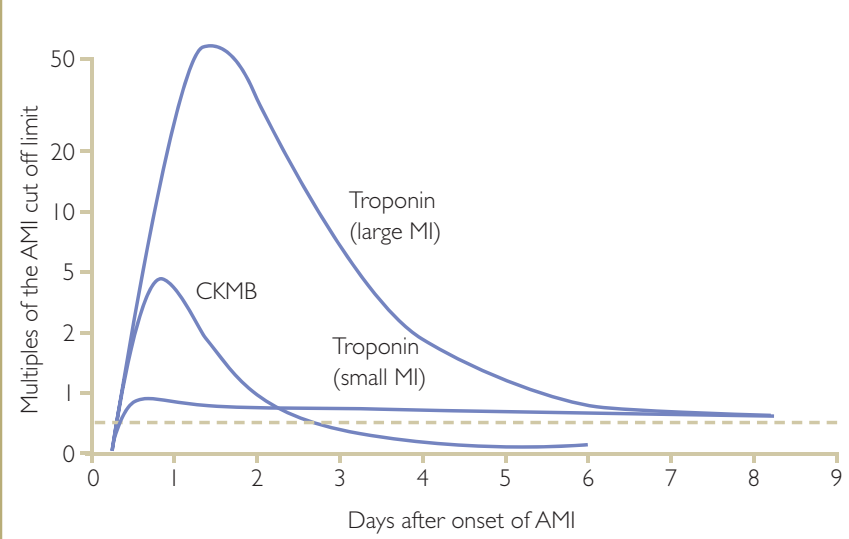

The broken line reflects the 99th percentile with a coefficient of variation less than $10 \%$.

Figure is based on the Universal Definition of Myocardial Infarction Eur Heart J 2007; 28:2525-2538.

level defined as abnormal be raised to that value at which a specific assay has a CV of 10 percent of less. ${ }^{(9)}$ Currently, it is recommended that the cut-off (clinical discriminator) value of Troponin $T$ or I used for the definition of $\mathrm{AMl}$ is $0.1 \mathrm{ng} / \mathrm{mL}$ according to $\mathrm{ROC}$ analysis.

\section{Use for diagnosis of AMI}

Cardiac troponin concentrations usually begin to rise 2 to 3 hours after the onset of AMI (Figure 2), with up to $80 \%$ of patients having elevated levels within this time frame. ${ }^{(10)}$ Troponin data is, therefore, additive to ECG data and superior to CK-MB results. Patients with ST-elevation myocardial infarction (STEMI) are different from those with unstable angina (UA) and non ST-elevation myocardial infarction (NSTEMI) in that they do not need biomarker measurements before institution of reperfusion therapy. However, STEMI patients presenting with elevated troponin on arrival have a lower rate of coronary recanalization, both with thrombolysis and direct percutaneous coronary intervention (PCl) and have adverse short and long-term prognosis. ${ }^{(11)}$ In patients without diagnostic ST-segment elevation, serial troponin testing should be performed after 6 hours if the initial values are indeterminate to diagnose or exclude AMI with high accuracy.

Elevations in cardiac troponins after AMI persist for up to 10 days, permitting late diagnosis. ${ }^{(12)}$ In contrast, CK-MB elevations return to baseline levels by 36 to 48 hours and cannot be used for late diagnosis. Previously, new elevations in CK-MB were used to detect reinfarction. Troponins can now be used for this purpose, bearing in mind that a $20 \%$ increase from prior levels is supportive for the detection of ongoing ischemia (Figure 3).
FIGURE 3: Algorithm for diagnosis of suspected early recurrent $M$ (reinfarction) after STEMI based upon clinical setting and troponins (tn)*

Clinical setting
of Chest Pain

It is important to note that, while troponin measurement is a useful tool for the diagnosis of ACS, several limitations exist. Since troponin concentrations only begin to rise 2 to 3 hours after the onset of $\mathrm{AMI}$ patients who present earlier will have negative troponin levels, thereby limiting the diagnostic capabilities of this biomarker. Certain patients with unstable angina present with acute chest pain and myocardial ischemia, and yet have negative troponin levels. It has been shown that these patients still have a high incidence (6-8\%) of AMI at 30 days, even with the use of state of-the-art evidence-based therapy. ${ }^{(13)}$ Similarly, a subset of patients with ACS may not develop necrosis despite severe coronary artery disease and myocardial ischemia. They clearly need further characterization and advice but escape scrutiny due to negative troponin results. Owing to their tissue specificity, another limitation of troponins is their inability to discriminate between ischemic and nonischemic myocardial injury, such as traumatic injury, congestive heart failure, pericarditis, and myocarditis. ${ }^{(14)}$

\section{Prognostic Value}

Troponin elevation has been shown to correlate with the severity of coronary disease and long-term mortality in patients presenting with STEMI and NSTEMI. A pooled analysis of 21 studies involving 18982 patients with ACS found that elevated troponins were also associated with an increased risk of cardiac death or reinfarction at 30 days (odds 
ratio $3.44,95 \% \mathrm{Cl} 2.94$ - 4.03).(15) High-risk ACS patients with elevated troponin do better with early invasive therapy, glycoprotein I l b/ I I la inhibitors, anti-platelet agents and low molecular-weight heparins. ${ }^{(9)}$ Thus, the impact of a positive or negative troponin at presentation would seem to be central to the management decision. The degree of elevation of troponin also has significant prognostic value, with the risk of death increasing proportionately with the absolute troponin level. ${ }^{(16)}$

\section{CK- MB ISOENZYME}

Since $C K$ is widely distributed in tissues, elevations in total serum $C K$ lack specificity for cardiac damage, and a consensus statement of the European Society of Cardiology and the America College of Cardiology concluded that total CK no longer has a role in diagnosing an AMI. ${ }^{(5)} \mathrm{It}$ has been recommended that the biochemical diagnosis of AMI be based upon the rise and/or fall in CK-MB when troponin assays are not available. CK-MB typically begins to rise 4 to 6 hours after the onset of infarction and returns to baseline within 36 to 48 hours (Figure 2) Most assays for CK-MB measure CK-MB mass; such assays are more sensitive than active assays. Because CK-MB comprises a lower fraction in skeletal muscle than in the heart, percentage criteria (from 2.5 to 5 percent) have been proposed to distinguish skeletal muscle damage from cardiac damage. These criteria are not recommended, since they improve specificity, but do so at the cost of sensitivity in patients who have both skeletal and cardiac injury. ${ }^{(7)}$

Typical criteria for the diagnosis of an AMI require a total CK elevation twofold above normal with a simultaneously elevated CK-MB. However, ischemic injury may occur in the presence of a normal total CK but elevated CK-MB. In a review of almost 26000 patients with NSTEMI , the primary endpoint of death or AMI at 180 days was significantly higher in patients with elevated CK-MB, independent of whether total CK was normal or elevated. ${ }^{(17)}$

\section{MYOGLOBIN}

The ubiquitous heme protein myoglobin is elevated in roughly similar proportions as CK-MB and troponins among patients with AMI. ${ }^{(18)}$ However, several limitations exist to the use of serum myoglobin for the diagnosis of AMI:

- The rapid release and metabolism of myoglobin can result in an undulating pattern characterized by increases and decreases in serum myoglobin concentration that can lead to clinical confusion. ${ }^{(19)}$
Myoglobin also lacks specificity for the heart and serum concentrations are elevated after injury to a variety of tissues, particularly skeletal muscle. Myoglobin levels are also increased following recent cocaine use and in patients with impaired renal function due to decreased clearance. ${ }^{(20)}$

Because of these limitations and absence of any advantage over troponins, myoglobin should not be routinely measured in patients with suspected AMI.

\section{HIGH-SENSITIVITY C-REACTIVE PROTEIN (HSCRP)}

The acute phase reactant CRP is perhaps the best characterized of the currently available inflammatory biomarkers. Clinically, CRP can be measured with several standardized, validated, and inexpensive highsensitivity assays. ${ }^{(21)}$ Several prospective, epidemiological studies have demonstrated that hsCRP is an independent predictor of risk of AMI, stroke, peripheral arterial disease, and sudden death, even in apparently healthy individuals. ${ }^{(22)}$ Levels of hsCRP of $<1 \mathrm{mg} / \mathrm{ml}$, I to $3 \mathrm{mg} / \mathrm{ml}$, and $>3 \mathrm{mg} / \mathrm{ml}$ should be interpreted as lower, moderate and higher vascular risk, respectively. Any clinical use of hsCRP is best limited to those at intermediate risk, that is individuals with anticipated 10 -year event rates between $6 \%$ and $20 \%$

There is no evidence yet that lowering hsCRP will necessarily lower vascular risk. Furthermore, there are still ongoing debates about the stability of CRP in stored (frozen) samples of plasma or serum, as well as about its biological intra-individual variability. (23) One ongoing, prospective, randomized study being conducted in apparently healthy individuals (the Use of Statins in Primary Prevention: An Intervention Trial Evaluating Rosuvastatin (JUPITER) study) is evaluating whether hsCRP-guided statin administration will have a favorable impact on cardiovascular disease. Thus, hsCRP may yet be proved to be a biomarker able to guide primary prevention studies.

\section{B-TYPE NATRIURETIC PEPTIDES}

Brain natriuretic peptide (BNP) and its N-terminal amino acid fragment (NT-proBNP) are neurohormones synthesized predominantly in the ventricles and released into the circulation in response to ventricular dilation and pressure overload. These peptides have been found to be useful in assisting with the diagnosis of congestive heart failure in dyspneic patients, with increasing levels correlating grossly with the severity of left ventricular dysfunction (LVD), both clinically and haemodynamically. ${ }^{(24)}$ More recently, multiple large studies have shown BNP and NT-proBNP levels to be strong independent predictors of LVD and death in the months after both STEMI and NSTEMI. ${ }^{(25)}$ It has 
also been demonstrated that NT-proBNP is able to define not only myocardial necrosis, but also reversible ischemic changes in patients with $\mathrm{ACS}$. ${ }^{(26)}$

The mechanisms for the increase in NT-proBNP in patients with ACS and normal ejection fraction have not been precisely defined. It has been suggested that cardiac myocytes secrete natriuretic peptides during the prolonged ischemic phase that precedes cell death. ${ }^{(26)}$ Therefore, ischemia may be an additional stimulus for NT-proBNP synthesis. ${ }^{(27)}$

Furthermore, serial measurements of NT-proBNP may permit an ongoing assessment of risk in patients with ACS. Since this peptide is highest during the acute phase of presentation and then gradually falls over 6 months, elevated levels at any time period are associated with increased risk. ${ }^{(28)}$ This biomarker rises rapidly during the first 24 hours following an AMI and may be considered as an additional marker in the diagnosis of $\mathrm{AMI}$, especially in those without regional wall motion abnormalities on echocardiography. ${ }^{(29)}$

Although B type natriuretic peptides are promising across the entire spectrum of ACS, they are still in the early stages of widespread clinical use. There is a pressing need to address several assay-related issues, their application to different ethnic groups, and finally the costeffectiveness of these peptides before they are used on a large-scale clinical and epidemiological basis.

\section{OTHER NOVEL MARKERS}

A variety of other biomarkers have been evaluated in the context of ACS, but none are available for routine clinical use.

- Heart-type fatty acid binding protein (H-FABP) is a small cytosolic protein that behaves similarly to myoglobin in its kinetics and release. It is released into the circulation in response to myocardial injury, and may provide added value to other cardiac biomarkers for the early diagnosis of $\mathrm{AMI}{ }^{(30)}$

- The albumin cobalt binding test, which measures the concentration of ischemia-modified albumin (IMA), has been recently proposed for early detection of myocardial ischemia without infarction. ${ }^{(31)}$ The main limitation of IMA at present is its low specificity, since the test is positive in many conditions like cancer, infections, end-stage renal disease, liver disease and brain ischemia. In addition, the need for adjustment for baseline albumin, and the frequent overlap between normal and abnormal values, renders this test difficult to interpret in many patients.
The pregnancy-associated plasma protein-A (PAPP-A) is a zincbinding matrix metalloproteinase, which is expressed in eroded and ruptured atherosclerotic plaques. The PAPP-A level has been recently acknowledged as a strong predictor of cardiovascular events in patients with ACS. ${ }^{(32)}$

- Increasing evidence suggests that soluble CD40 ligand plays an important part in disease progression and plaque destabilization. ${ }^{(33)}$ Soluble CD40 ligand, which is expressed on platelets and released from them on activation, has been found to be a powerful biomarker of inflammatory thrombotic activity in patients with ACS. ${ }^{(34)}$ Elevated levels of this biomarker can also identify patients with ACS who are at highest risk for cardiac events. Therefore, soluble CD40 ligand not only contributes to the pathophysiology of ACS but also represents a reliable and powerful clinical marker for use in identifying patients with high-risk atherosclerotic lesions, coronary thrombosis, or both. ${ }^{(35,36)}$

- A promising new biomarker is growth differentiation factor I5 (GDF-15), a member of the growth factor beta cytokine superfamily. GDF- 15 is released from myocardial cells after ischemia and reperfusion injury and has recently been shown that circulating levels are a strong independent marker of 1 -year mortality in patients with acute coronary syndrome. ${ }^{(37)}$

- Several other markers of inflammation, which may show clinical promise, include lipoprotein-associated phospholipase A2, also known as platelet-activating factor acetylhydrolase, ${ }^{(38)}$ tumor necrosis factor alpha, ${ }^{(39)}$ myeloperoxidase, ${ }^{(40)}$ interleukin-6 and 8 , and the leucocyte adhesion molecules ICAM-I, VCAM-I, and P-selectin. ${ }^{(41)}$

\section{CONCLUSIONS}

Although this overview has focused on the role of several cardiac biomarkers in ACS, it is important to recognize that the fundamental issue with all markers is that there is no gold standard for the diagnosis of cardiac ischemia. Everyone is interested in finding the next biomarker that will be more specific and possibly cheaper than the current biomarkers in the diagnosis and risk stratification of ACS. Perhaps, ongoing efforts in plasma-based biomarker research should concentrate on the use of a multimarker approach to enhance our diagnosis, prognostic assessment and management of patients with suspected ACS, as compared with using individual markers alone. It is recommended that current practice involve the selective use of biomarkers in patients in whom a more complete assessment of risk is desired. At present, routine biomarker measurement is not advocated until further data become available, which will support the benefit of specific therapeutic interventions. 


\section{REFERENCES:}

I. Lampe FC, Morris RW, Walker M, et al. Trends in rates of different forms of diagnosed coronary heart disease, 1978 to 2000: prospective, population based study of British men. BMJ 2005; 330: 1046

2. Rosalki SB, Roberts R, Katus HA, et al. Cardiac biomarkers for detection of myocardial infarction: perspectives from past to present. Clin Chem 2004: 50:2205-13.

3. Fox KAA, Birkhead J, Wilcox R, et al. British Cardiac Society Working Group on the definition of myocardial infarction. Heart 2004; 90:603-9.

4. Karras DJ, Kane DL. Serum markers in the emergency department diagnosis of acute myocardial infarction. Emerg Med Clin North Am 200 I; 19:321-37.

5. Myocardial Infarction Redefined - A Consensus Document of the Joint European Society of Cardiology / American College of Cardiology Committee for the Redefinition of Myocardial Infarction. J Am Coll Cardiol 2000; 36:959-68.

6. Morrow DA, De Lemos JA. Benchmarks for the Assessment of Novel Cardiovascular Biomarkers. Circulation 2007; 1 15:949-52.

7. Adams JE 3rd, Abendschein DR, Jaffe AS. Biochemical markers of myocardial injury. Is MB creatine kinase the choice for the 1990s? Circulation 1993; 88:750-63.

8. Katus HA, Remppis A, Scheffold T, et al. Intracellular compartmentation of cardiac troponin $T$ and its release kinetics in patients with reperfused and nonreperfused myocardial infarction. Am J Cardiol 1991; 67:1360-7.

9. Braunwald $\mathrm{E}$, Antman EM, Beasley JW, et al. ACC/AHA guidelines for the management of patients with unstable angina and non-ST-segment elevation myocardial infarction: a report of the American College of Cardiology / American Heart Association Task Force on Practice Guidelines. J Am Coll Cardiol 2000; 36:970-1062.

10. Macrae AR, Kavsak PA, Lustig V, et al. Assessing the requirement for the 6-hour interval between specimens in the American Heart Association Classification of Myocardial Infarction in Epidemiology and Clinical Research Studies. Clin Chem 2006; 52:8I2-8.

II. Giannitsis E, Muller-Bardorff M, Lehrke S, et al. Admission troponin T level predicts clinical outcomes, TIMI flow, and myocardial tissue perfusion after primary percutaneous intervention for acute ST-segment elevation myocardial infarction. Circulation 200 I; 104:630-35.

12. Christenson RH, Ohman EM, Topal EJ, et al. Assessment of coronary reperfusion after thrombolysis with a model combining myoglobin, creatine kinase-MB, and clinical variables. TAMI-7 Study Group. Thrombolysis and Angioplasty in Myocardial Infarction - 7. Circulation 1997; 96:1776-82.

13. Ridker PM, Brown NJ,Vaughan DE, et al. Established and emerging plasma biomarkers in the prediction of first atherothrombotic event. Circulation 2004; I09:IV6-IVI 9.

14. Panteghini M. Role and importance of biochemical markers in clinical cardiology. Eur Heart | 2004; 25: 1 187-96.

15. Ottani F, Galvani M, Nicolini FA, et al. Elevated cardiac troponin levels predict the risk of adverse outcome in patients with acute coronary syndromes. Am Heart J 2000; I 40: $917-27$.

16. James S, Armstrong P, Califf R, et al. Troponin T levels and risk of 30-day outcomes in patients with the acute coronary syndrome: prospective verification in the GUSTO-IV trial. Am J Med 2003; 1 I5:178-84

17. Galla JM, Mahaffey KW, Sapp SK, et al. Elevated creatine kinase-MB with normal creatine kinase predicts worse outcomes in patients with acute coronary syndromes: results from 4 large clinical trials. Am Heart J 2006; 151:16-24.

18. Zimmerman J, Fromm R, Meyer D, et al. Diagnostic Marker Cooperative study for the diagnosis of myocardial infarction. Circulation 1999; 99:1671-7.

19. Kagen L, Scheidt S, Butt A. Serum myoglobin in myocardial infarction: The "staccato phenomenon." Is myocardial infarction in man an intermittent event? Am J Med 1977; 62:86-92.

20. Roberts R, Myoglobinemia as an index to myocardial infarction. Ann Intern Med 1977; 87:788-9.

21. Ledue TB, Rifai N. Preanalytic and analytic sources of variations in C-reactive protein measurement: implications for cardiovascular disease risk assessment. Clin Chem 2003; 49:1258-71.

22. Torres JL, Ridker PM. Clinical use of high-sensitivity C-reactive protein for the prediction of adverse cardiovascular events. Curr Opin Cardiol 2003; | 8:47| -78.
23. Bogaty $\mathrm{P}$, Brophy JM, Boyer L, et al. Fluctuating inflammatory markers in patients with stable ischemic heart disease. Arch Intern Med 2005; 165:221-26.

24. Maisel A, Krishnaswamy P, Nowak R, et al. Rapid measurement of B-type natriuretic peptide in the emergency of diagnosis of heart failure. N Engl J Med 2002; 347: |61-67.

25. Wiviott S, De Lemos J, Morrow D. Pathophysiology, prognostic significance and clinical utility of B-type natriuretic peptide in acute coronary syndromes. Clin Chim Acta 2004 346:1 19-28.

26. Goetze JP, Christoffersen C, Perko M, et al. Increased cardiac BNP expression associated with myocardial ischemia. FASEB 2003; 17:1 105-7.

27. Ranjith N, Pegoraro RJ, Naidoo DP, et al. Prognostic value of N-terminal-pro-brain natriuretic peptide measurements in patients with acute coronary syndromes. Cardiovasc J South Afr 2006; 17:60-6.

28. Lindahl B, Lindback J, Jernberg T, et al. Serial analyses of $\mathrm{N}$-terminal pro-B-type natriuretic peptide in patients with non-ST-segment elevation acute coronary syndromes: a Fragmin and fast Revascularization during In Stability in Coronary artery disease (FRISC)-II substudy. J Am Coll Cardiol 2005; 45:533-4I.

29. Ranjith N, Pegoraro RJ, Naidoo DP, et al. The Role of Echocardiography and its Comparison with NT-proBNP Measurements in Patients with Acute Myocardial Infarction. Med Sci Monit 2007; 1 3:574-8.

30. Seino Y, Ogata K, Takano T, et al. Use of a whole blood rapid panel test for the hearttype fatty acid-binding protein in patients with acute chest pain: comparison with rapid troponin T and myoglobin tests. Am J Med 2003; I I5:185-90.

31. Christenson RH, Duh SH, SanhaiWR, et al. Characteristics of an Albumin Cobalt Binding Test for assessment of acute coronary syndrome patients: a multicenter study. Clin Chem 200।; 47:464-70.

32. Heeschen C, Dimmeler S, Hamm CW, et al. CAPTURE Study Investigators: Pregnancyassociated plasma protein-A levels in patients with acute coronary syndromes: comparison with markers of systemic inflammation, platelet activation, and myocardial necrosis. J Am Coll Cardiol 2005; 45:229-37.

33. Mach F, Schonbeck U, Sukhova GK, et al. Reduction of atherosclerosis in mice by inhibition of CD40 signalling. Nature 1998; 394:200-203.

34. Heeschen C, Dimmeler S, Hamm CW, et al. Soluble CD40 Ligand in Acute Coronary Syndromes. N Engl | Med 2003; 348: I 104- I I II.

35. Andre P, Prasad KS, Denis CV, et al. CD40L stabilizes arterial thrombi by a beta3 integrin-dependent mechanism. Nat Med 2002; 8:247-252.

36. Andre P, Nannizzi-Alaimo L, Prasad SK, et al. Platelet-derived CD40L: the switch-hitting player of cardiovascular disease. Circulation 2002; 106:896-899.

37. Wollert KC, KempfT, PeterT, et al. Prognostic value of growth-differentiation factor-15 in patients with non ST-elevation acute coronary syndrome. Circulation 2007; 1 |4: 550-7.

38. Garza CA, MontoriVM, McConnell JP, et al. Association between lipoprotein-associated phospholipase A2 and cardiovascular disease: a systematic review. Mayo Clin Proc 2007; 82:159-165.

39. Pai JK, Pischon T, Ma J, et al. Inflammatory markers and the risk of coronary heart disease in men and women. N Engl J Med 2004; 351:2599-2610.

40. Brennan ML, Penn MS, Van Lente F, et al. Prognostic value of myeloperoxidase in patients with chest pain. N Engl J Med 2003; 349: 1595-I 604.

41. Hartford M, Wiklund O, Mattsson H, et al. C-reactive protein, interleukin-6, secretory phospholipase A2 group IIA and intercellular adhesion molecule-I in the prediction of late outcome events after acute coronary syndromes. Journal of Internal Medicine 2007; 262:526-536. 